\title{
The Structure Design and Analysis of Hydraulic Power Unit Li Chang
}

\author{
Shandong vocational college of light industry, Shandong, Zbo, 255300, China.
}

\author{
Keywords: Hydraulic pressure; Hydraulic components; Layout; Design
}

\begin{abstract}
In this paper, the structural design of a hydraulic power unit is analyzed. Firstly, the hydraulic transmission is introduced, and then the innovation and development of hydraulic technology are analyzed from two aspects of hydraulic field bus technology and hydraulic pressure system. The structure of the hydraulic power unit is analyzed, including three kinds of layout, layout scheme and fuel tank design. Finally, the overall structural design is analyzed.
\end{abstract}

\section{Introduction}

In recent years, due to the application of the electronic technology, computer technology, information technology, automatic control technology and new technology, new materials, fluid power transmission has new development. Pneumatic and hydraulic systems and components in technical level have been greatly improved. It has tumed in to an industrial machinery. Engineering construction machinery and the important technology of the national defense tip products. And its automation, high accuracy, high efficiency, high speed, high power, miniaturization and lightweight are the key to continuously improve the competitiveness of electric and mechanical transmission.

\section{Basic introduction of hydraulic drive}

Hy draulic tran smission is a liquid as the working medium, the use of pressure can drive the actuator transmission mode. In the hydraulic system of the machine tool table driven by tank, a filter, a hydraulic pump, a relief valve, stop valve, throttle valve, reversing valve, hydraulic cylinder and the connecting these components of the tubing, fittings and other components.

1) The work principle is as follows: the motor drives the hydraulic pump through the oil filter from the oil tank. The oily fluid is pressurized, from the pump output port input line. The oil is driven by opening stop valve, throttling valve, reversing valve into the hydraulic cylinder, and the piston is driven to move the bench or so. The oily fluid in the hydraulic cylinder is discharged back to the tank by reversing valve and return line.

2) The speed of the operating table is adjusted by the throttle valve. When the throttle valve is opened, the amount of oil entering the hydraulic cylinder increases, the speed of the operating table is increased, when the throttle valve is shut off, the oil quantity of the hydraulic cylinder is reduced, and the moving speed of the working table is reduced. Thus, the speed depends on the amount of oil.

\section{Innovation and development of hydraulic technology}

Hydraulic field bus technology. The characteristics of field bus technology in the application of hydraulic system.

1) Economy. Any new technology, the development and use of innovative products, the cost is one of the first factors to consider, bus technology is no exception. One of the original intentions of designing and developing the bus technology is to reduce the system and the engineering cost. So, the first premise that the application unit uses the bus product and the supplier to provide the product should be the purpose of reducing the user cost of the bus system.

2) E C I, 61131 - 3 standard flexible procedures is easy to learn, to understand, and it is operability and strong. 
3) Reliability and maintainability.

4) Friendly man-machine dialogue interface can be easy for the hydraulic system parameter modification and fault monitoring.

5) Meet all the important criteria for personal safety, electromagnetic compatibility, impact resistance and shock resistance.

6) Compared with the traditional hydraulic proportional control system, it has its price competitive advantage.

Hydraulic components and systems. Hydraulic transmission technology features

1) Resource rich, extensive sources, and high utilization rate. Water is the most abundant and symbiotic resource on earth, and can be recovered repeatedly in the whole cycle of the application of a hydrostatic transmission system, and it is not easy to deteriorate.

2) Water is a kind of non-toxic and pollution-free resource, especially it has no harm to human body and environment. To improve the comfort and safety of the environment, the liquid without any treatment can be directly discharged. Fundamentally eliminate hydraulic transmission system Gang leaks and emissions are caused by cattle production and environmental pollution.

3) The safety of excellent flame retardant is high. Application is especially suitable for high temperature, nuclear radiation, fire and other occasions, effectively it can solve hydraulic trans mission of flammable, explosive, oil vapor on the human body warm security and nuclear radiation caused by oil metamorphism and radioactive pollution problems.

4) The technology and process of the processing are simple. The operation and maintenance of the system is low. Using water for a long time is not bad, and the treatment of water before and after the use is straightforward; and work in water environment system in shipping, underwater operations, submarine, with out tank, cooling device, greatly simplifies the system.

Application and Prospect of hydrostatic tran smission technology

With the progress of science and technology, hydraulic products and technology has made great progress, at present, not only the water pump form increased in line with the ISO / setup connecting various valves of the ruler of the stand ard specifications, even Superposition valve, proportional valve and continuously adjustable flow control valve formed a series of products, supporting the use of the hydraulic cylinder, fuel tank, joint parts, seals, etc. also should there be one, are under the guidance of experts. Users can distribute the ststem groups according to their own needs. A variety of operating pressure was officially launched for the L6 21MPa as a complete set of machinery and equipment used by the independent products (power station and a control valve). The hydraulic pressure transmission is the old technology which rises in the first industrial revolution, and through the innovation development, it will become fourth kind of drive technology which is the electrical, oil pressure, the pneumatic movement.

\section{Structural analysis of hydraulic power unit}

According to the arrangement of a hydraulic power source is divided into upper, non portable and cabinet three.

Comparison of various arrangement. Hy draulic power source of the SRE covers an area of small, compact structure, hydraulic pump arranged in the oil tank in the vertical installation of power source with the advantages of low noise and being easy to collect oil spills, are commonly used in small and medium power hydrau lic pump station. When using horizontal power source, due to that the hydraulic pump is arranged on the tank above photosphere, all kinds of hydraulic pump suction height must be paid attention to, to anti hydraulic pump oil outlet with too high vacuum degree, resulting in sucking air or cavitation.

Non home hydrau lic power source due to hydraulic pump arranged in the oil tankbelow the surface of the liquid, so it can effectively improve the suction capacity of hydraulic pump. The power source installation height is low and is easy to maintain, but covers a large area on pump inhalation which 
allows the height limit, transmission power is larger, and the space is not restricted, and starting rate is low, when in use, it is quickly put into operation occasions, which can become a whole set of hydraulic pumps, the fuel tank components, control components, such as sets of hydraulic power source of a new generation of this arrangement, mechanism is quite compact, and it can guarantee a certain pressure and displacement, such as in recent years the general strong hydraulic power unit is typical representative.

Cabinet type hydraulic power source device can be at the top of the cabinet body and arrangement of all kinds of instrument board and an electric control box, and the appearance is tidy and beautiful, because the pump group is enclosed switchgear and it can shield noise, and can effectively reduce the pollution of the outside world, the drawback is due to the heat dissipation of the need which takes account of the operation and maintenance of space and ahydraulic system, resulting in the overall size of the larger ones, it usually only used in the middle and small power applications and laboratory.

Selection of layout scheme. In view of the design of hydraulic power source of the actual use of the environment, under the premise of meeting the requirements of pressure and displacement, the system do the smaller and more compact and better, which has the advantages of small occupied area, saving many materials, in this premise, in the size and layout to the hydraulic power unit formnear.

Because of the corrosive nature of seawater, the most contact parts of the hydraulic power unit of the hydraulic power element should use special materials. Here the motor and hydraulic pumps in the internal tank. Change the motor is in a more advanced underwater motor. Two methods are adopted for the stress of the water pressure of the motor and the isolation of the water.

- mechanical structure of water pressure: the use of high mechanical strength of the material to increase the wall thickness of the casing to withstand the pressure and the isolation of seawater.

- equilibrium pressure of structure: inside the motor oil filled, non metal membrane box is to be closed, by the expansion and contraction of the box this film adaptation within the fluid thermal expansion and changes in the external water pressure, motor inside and outside pressure equilibrium is reached.

Fuel tank design. Generally, the tank can be divided into the overall type of fuel tanks, dual-use tanks and independent fuel tanks three categories.

- Monolithic fuel tank is the fuel tank formed in the hydraulic system or the components of the machine.

- dual fuel tank is the hydraulic oil and other purposes in the machine to the oil tank.

- independent fuel tanks are the most widely u sed fuel tanks.

Tank capacity of the system is calculated according to the empirical formula, because when the system is working, the tank is immersion in seawater and sea temperature generally about 4DEGC, so radiating habits of the whole system is very good, the equivalent of cold water, there is no need to calcu late the volume of oil from the aspect of heat dissipation.

The empirical formula for fuel tank capacity is (formula 1):

$\mathrm{V}=a Q_{v}$

Hydraulic pump flow; ---- empirical coefficient, in general, from 3 to 5 . The volume $\mathrm{V}$ of the oil needed for the system is 157.5 (L).

In this paper, the design of the system structure is the hydraulic pump and other parts of the placed inside the tank, so these parts of the volume and the volume of oil required tank volume calculation are needed. The shape of the hydraulic pump is highly irregular, to calculate the accurate the size is very difficult. In this paper, the size is approximately considered as a cube, the size of each direction folded is $195 \mathrm{~mm} * 202 * 233 \mathrm{~mm}$. The volume of the hydraulic pump is: $9.18 * 10$ hydraulic pump volume and other parts of the volume, the final total volume of the round is $9.2 \times 10$, that is, $9.2 \mathrm{~L}$. The volume of the entire fuel tank is $1.667 * 10$.

Selected deep-sea DC motor with a diameter of about $400 \mathrm{~mm}$, in order to ensure the appearance of the whole system, but also to en sure the whole device not for too long, end diameter of the tank should be $560 \mathrm{~mm}$, srefer to the length of the calculation of the volume of the tank. 
Overall system structures. Determine the diameter and length of the tank, and determine the thickness of the tank. Although the tank with the pressure balance device is in the deep sea to withstand the pressure differential of approximately zero, it still need to bear greater pressure, at the same time, it also plays many threaded holes for the other components are mounted and fixed, so tank wall thickness should be $10 \mathrm{~mm}$. Tank manufacturing method is selected in casting process. In order to make the system be easy to in stall, the right end face of the oil tank is opened, after the installation of the internal structure with a cover plate of the tank and the tank cover, in order to facilitate filling the whole system, at the top of the tank wall is opened a oiling. The use of storage is bag shaped structure as the pressure balance structure, taking into account the convenient installation, put them in the tank on the back of, but the system in the initial state of the airbag can be filled with oil, air bag structure, the highest position is lower than the highest position of the tank wall.

\section{Conclusions}

Hydraulic power transmission has a large power density or force density. Force density here refers to work pressure. Hydraulic device is easy to speed stepless adjustment, and the speed is range. The speed adjustment can also be in the work process. Hydraulic device works smoothly with small impact and is easy to achieve frequent commutation.

Hydraulic device is easy to overload protection, which can can achieve self - lubrication with long service life. Hydraulic device is easy to realize automation and can be conveniently on the liquid flow direction, pressure and flow of regulation and control, and can easily and electrical, electronic control and pneumatic control combination, the realization of complex movement and operation. Hydraulic components are easy to achieve serialization, standardization and generalization, which is easy to design, manufacture and promote the use. The development of the hydraulic transmission device in Chin a has considerable potential.

\section{References}

[1] Tianjue Lei. A new hydrau lic eng ineering handbook. Beijing: Beijing Institute of Technology press, 1998.

[2] Committee of Chinese Mechanical Engineering Society of China Mechanical Design Canon. Jian yun Li. China Mechanical Design Can on brother 5 volume designs of mechanical control system. Nanchang: Jiangxi science and Technology Press, 2002.

[3] Japan hydraulic pneumatic Association. Hydraulic pneumatic manual. Beijing: Mechanical Engineering Press, 1984.

[4] Qibai Li. Handbook of hydraulic components. Beijing: Metallurgical Indu stry Press, 2000.

[5] Tiehua Zhang, Cunxing He. Hydraulic and pneumatic transmission. Wuhan: Huazhong University of Science and Technology press, 2000.

[6] Jihai Jiang, hydraulic transmission. Harbin: Harbin Institute of Technology press, 1997.

[7] Mingren Xiong, Huixiong Wang. Hydraulic and pneumatic transmission. Beijing: National Defense Industry Press, 2003.

[8] Zhongfan Guan. The hydraulic drive sy stem, Beijing: Mechanical Industry Press, 1998.

[9] Jianmin Zuo . Hydraulic and pneumatic transmission. Third ed. Beijing: mechanical industry, 2005.

[10] Mingxin Jia, hydraulic transmission and control. Beijing: National Defense Industry Press, 2001. 